\title{
Mesh Migration in to Colonic Lumen Post Abdominal Hernia Repair: A Case Report
}

\author{
Ahmed Kharief* \\ Surgical Department, Louth County Hospital, Ireland \\ *Corresponding author: Ahmed Kharief, Surgical \\ Department, Louth County Hospital, 3 Ashfield Crescent \\ North Road Drogheda Co Louth, Ireland
}

Received: February 01, 2017; Accepted: February 17, 2017; Published: February 22, 2017

\begin{abstract}
Incisional hernia formation following secondary to abdominal surgeries is a common complication. Laparoscopic mesh repair recently has gained significant publicity for such hernia repair. However, utilizing polypropylene mesh for incisional hernia repair, can lead to variety of complications from minor postoperative hematoma and seroma to mesh rejection and fistula formation. However, mesh migration is an infrequent occurrence and has been rarely reported in the literature. Additionally, review of literature shows mesh migration to urinary bladder, scrotum and caecal lumen. We present a case of delayed partialtrans mural mesh migration from the abdominal wall into colonic lumen, following laparoscopic mesh repair of abdominal incisional hernia. This is the first case of mesh migration that has been successfully managed conservatively.

Keywords: Hernioplasty complication; Mesh migration; Cecum erosion; Chronic abdominal pain
\end{abstract}

\section{Case Report}

We present a 65-year-old, female, with background history of gallstone pancreatitis, open pancreatic necrosectomy and cholecystectomy 5years ago (2008), underwent open mesh repair for incisional hernia in August 2010 at another centre. The hernia was located at medial aspect of left sub-coastal (Kocher's) laparotomy scar. However, patient later developed incisional hernia at lateral aspect of the same laparotomy scar, for which she underwent Total Extra-Peritoneal hernia repair (TEP) at our surgical department in July 2011. Partietex composite mesh was applied to the $8 \times 8 \mathrm{~cm}$ hernial defect. Peri-operative course was uneventful. She re-attended surgical outpatient department in April 2014, with complains of ongoing right iliac fossa pain and bloating. She described the pain to be intermittent, dull ache, non-shifting, non-radiating, gradually worsening, with no associated aggravating or relieving factors. She denied any history of nausea, vomiting, diarrhoea, intermittent fever or per-rectal bleed. The pain was not related to food consumption or occurred at any particular timing. She had no recent weight loss. There was no similar history in the past. She did not have any significant family history of colorectal cancer. Her past medical history revealed left leg great saphenous varicose veins surgery (2002), colonoscopy showed sigmoid diverticulosis (2006), gall stones related pancreatitis (2007) large pancreatic pseudo cyst, followed by laparotomy with cholecystectomy and cystjujenostomy cyst drainage (2008). She is a non-smoker and consumed alcohol in moderation. Abdominal examination was unremarkable except for slight tenderness at right iliac fossa.

Radiology workup showed unremarkable plain abdominal-rays, ultrasound and Intravenous pyelogram. Patient was scheduled for colonoscopy on $16^{\text {th }}$ of July 2014. During the colonoscopy, a fixed foreign body appeared in the colonic lumen with apparent metallic tacker attached to its (Figure 1). Given her past medical history, mesh migration into colonic lumen was suspected. The defect was well sealed off and no signs of peritonitis or bleeding were apparent. However chronic inflammation around the mesh with fibrosis of the colonic mucosa was obvious (Figure 2). Patient underwent CTabdomen and pelvis, which showed foreign body in transverse colon, signifying the presence of intra-luminal mesh.

The above findings were disclosed to the patient and discussed that mesh migration often results in significant bleeding or a fistula and requires surgery. However, based on the patient's minimal symptoms, morbid obesity, and probability of significant surgical morbidity (postoperative wound infection, recurrent hernia, fistula, etc.), we suggested that the symptoms to be managed conservatively. The patient was discharged with instructions to return in case of significant bleeding or increased, disabling pain. She is attending regular follow-ups. On regular clinical follow-ups, patient is progressing well, with minimal symptoms and unremarkable examinations.

\section{Discussion}

Ventral hernias are the second most common type of hernia, second only to inguinal hernias. Historically, open Ventral Hernia Repair (VHR) had an over-whelming recurrence rate of $32-54 \%$, which only reduced by $10 \%$ after introduction of mesh in 1946. But this was burdened by complications such as postoperative hematoma and seroma, foreign body reaction, organ injury, wound infection, mesh rejection, and fistula/persistent draining sinuses. This was due to extensive dissection required for mesh placement. However, by advent of Laparoscopic Ventral Hernia Repair (LVHR), in 1993, not only the recurrence rate dropped to $4-16 \%$ but also the complications were minimised due to minimal tissue dissection [1]. Mesh repair is particularly important for incisional hernias with a diameter greater than $4 \mathrm{~cm}$ as the risk of recurrence is higher as the width increases.

Mesh migration following hernia repair is an uncommon complication. Moreover, migration to a completely intraluminal 


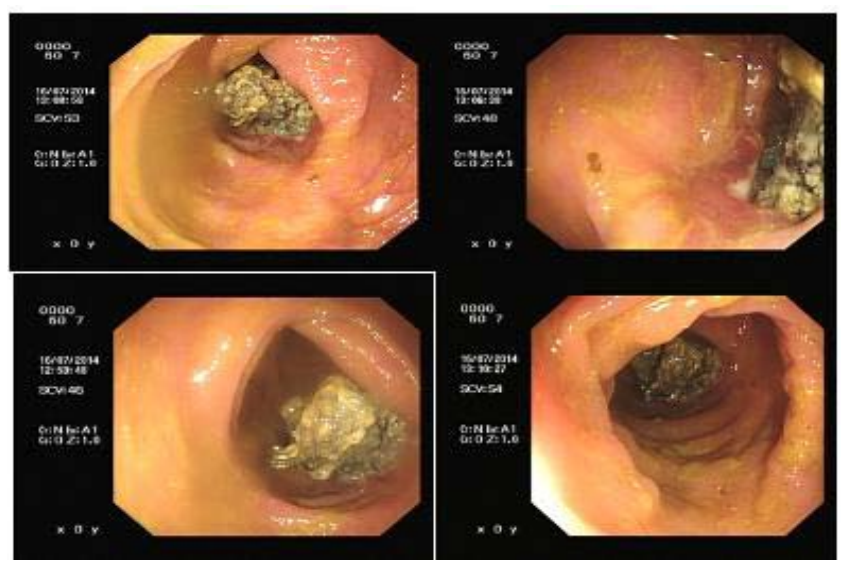

Figure 1: Presence of intra-luminal mesh complex.
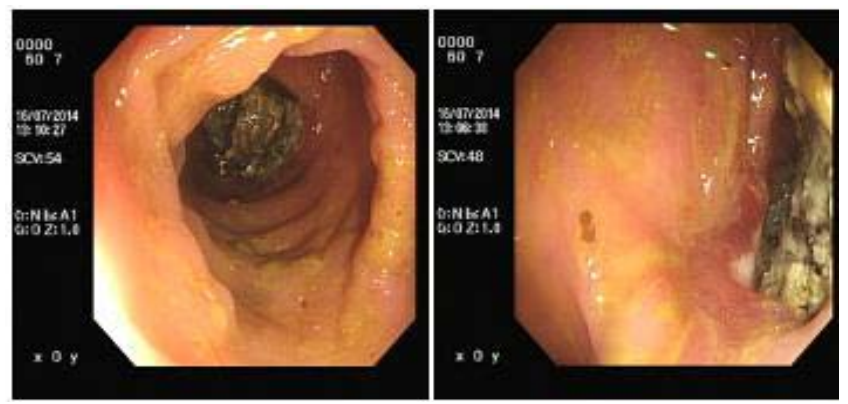

Figure 2: Signs of chronic inflammation.

position is exceedingly rare (Figures $3 \& 4$ ). Erosion into a viscous can be associated with migration or can occur due to mesh's original position. However, when erosion occurs, it can result in infection, abscess, fistula formation or intestinal obstruction.

Mesh migration occurs generally via two mechanisms. Primary mechanical migration occurs when an inadequately secured mesh traverses along adjoining paths of least resistance or when a relatively secure mesh is displaced by external forces [1]. Secondary migration, on the other hand, occurs through trans-anatomical planes and is the result of erosion triggered by foreign body reaction [2]. This mechanism has been supported by the presence of inflammatory granulation tissue at the site of migration. The latter process is gradual and may take several years. In our patient, the prolonged history and colonoscopy showing chronic inflammation around the mesh, confirmed it to be a secondary migration.

Mesh migration is rare and unpredictable. Clinical presentations are variable and related to the organ involved. Migration of knitted propylene mesh into the urinary bladder after laparoscopic left direct and indirect inguinal repair has been reported to cause haematuria $[3,4]$ and recurrent urinary tract infections. One report noted mesh plug migration into the scrotum after laparoscopic hernia repair that presented as a tender scrotal mass [5]. In another report involving scrotal migration of mesh, patient presented with strangulating bowel obstruction. The mesh had been placed via intra-abdominal approach during an emergency exploratory laparotomy [6]. Along with this, several reports of resultant enteric [7] and enterovesical fistulas [8]

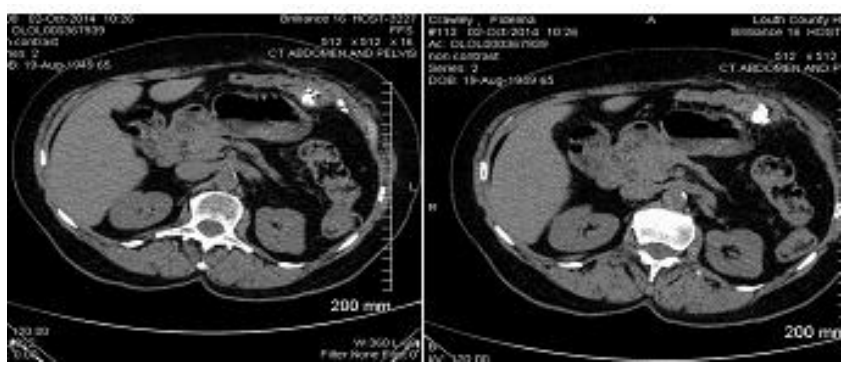

Figure 3: CT images showing mesh site at left flank with metallic tackers.

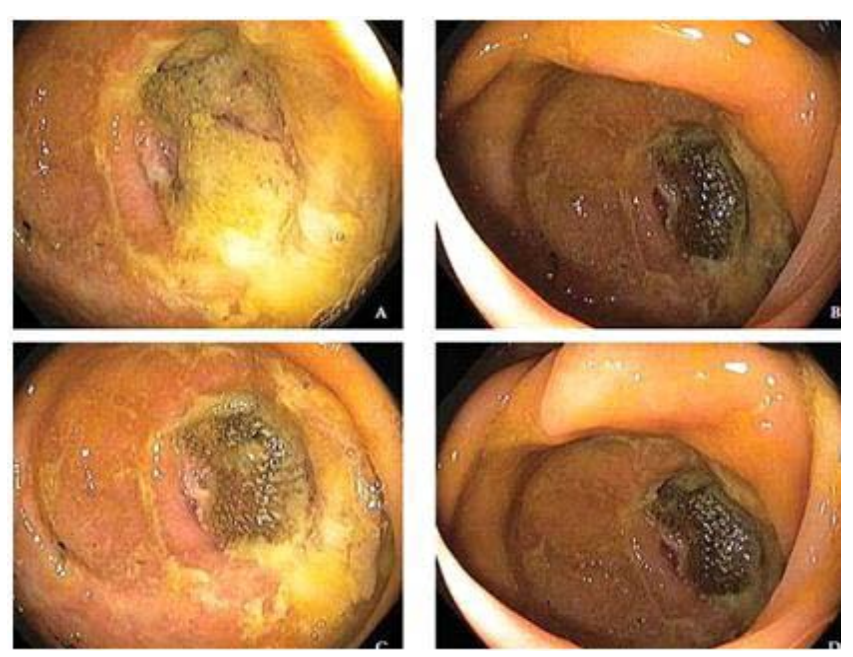

Figure 4: Presence of intra-luminal mesh in caecum.

have been reported. Successful colonoscopic removal of a migrated mesh from the colon at the splenic flexure has also been reported [3]. While the aetiology is unknown for mesh migration but based on the significant complications reported, the authors hypothesized that the method of fixation, as well as type of mesh, may have contributed to this problem.

The method of fixation may affect migration rates by altering the tensile strength and degree of movement of the mesh. However, the nature of the biomaterial is also important, as it affects the extent and degree of interaction with the surrounding tissue. Biological agents are being used with increasing frequency in abdominal wall hernias, where they have been shown to decrease foreign body reaction and potential complications secondary to infections. In a study regarding??[1] reported that $3 \%$ of patients with hernias repaired with a mesh plug suffer from morbidity due to migration of the plug. The size, shape, and positioning of the mesh may also be significant but unfortunately haven't been investigated previously. There are few cases reported of mesh migration into small bowel, bladder, large bowel, cecum, but all after laparoscopic repair of an inguinal hernia $[9,10]$. This is the first reported case of tension free open and laparoscopic mesh repair of an incisional hernia that resulted in mesh migration into the transverse colon.

Once there is an erosion of the mesh into the bowel, the question is whether or not it should be repaired. Repair would entail laparotomy, bowel resection, mesh resection, and anastomosis. 
However, there is a high likelihood of hernia recurrence after mesh removal, and placement of a mesh at the time of surgery may result in a high probability of postoperative infection. One solution would be a staged operation, with mesh/bowel resection first followed by a subsequent surgery to place a new mesh. Another would be to use biologic mesh at the time of bowel resection, but biological meshes are costly and are associated with complications such as stretching and may become infected.

Surgery would be a better choice for our patient as well but there were multiple risks to be encountered. First of all, she was morbidly obese and had multiple hernia repairs in the past, leading to a complicated surgery. Secondly, her mesh migration was in the right lower quadrant, a difficult area to repair hernias because mesh fixation around the iliac bone is difficult. Therefore after initial presentation and upon discussion of risks of surgery, with the patient, she was advised to have a regular follow up as she was minimally symptomatic from it and had no bleeding. If surgery were opted, the operation would have involved removing the mesh, right hemi colectomy, re-anatomizing of the bowel and then repairing the hernia with a biologic mesh.

In conclusion, mesh migration, particularly erosion, is a rare complication of any incisional hernia repair, especially when polypropylene mesh is used for repair. There is no clear cause of this complication, but new methods of mesh fixation, as well as types of mesh, are being investigated. It should also be recognized that mesh complications, particularly erosion, tend to occur years later and should be considered in atypical patient presentations. Tissue placement between the mesh and bowel to prevent direct contact of the two may help avoid this complication.
Given the popularity of these surgical procedures, complications may be frequently encountered. Gastroenterologists should thus be aware of the potential complications and the appropriate management.

\section{References}

1. Subramanian A, Clapp ML, Hicks SC, Awad SS, Liang MK. Laparoscopic ventral hernia repair: primary versus secondary hernias. Journal of Surgical Research. 2013; 181: e1-5.

2. Agrawal $A$, Avill R. Mesh migration following repair of inguinal hernia: a case report and review of literature. Hernia. 2006; 10: 79-82.

3. Celik A, Kutun S, Kockar C, Mengi N, Ulucanlar H, Cetin A. Colonoscopic removal of inguinal hernia mesh: report of a case and literature review. J Laparoendosc Adv Surg Tech A. 2005; 15: 408-410.

4. Hume RH, Bour J. Mesh migration following laparoscopic inguinal hernia repair. J Laparoendosc Surg. 1996; 6: 333-335.

5. Dieter RA. Mesh plugs migration into scrotum: a new complication of hernia repair. Int Surg. 1999; 84: 57-59.

6. Nowak DD, Chin AC, Singer MA, Helton WS. Large scrotal hernia: a complicated case of mesh migration, ascites, and bowel strangulation. Hernia. 2005; 9: 96-99.

7. Murphy JW, Misra DC, Silverglide B. Sigmoid colonic fistula secondary to Perfixplug, left inguinal hernia repair. Hernia. 2006; 10: 436-438.

8. Gray MR, Curtis JM, Elkington JS. Colovesical fistula after laparoscopic inguinal hernia repair. Br J Surg. 1994; 81: 1213-1214.

9. Goswarmi R, Babor M, Ojo A. Mesh erosion into caecum following laparoscopic repair of inguinal hernia (TAPP): a case report and literature review. J Laparoendosc Adv Surg Tech A. 2007; 17: 669-672.

10. Daas A, Matthew B, Elijah D, Kulkarni P. An unexpected mass: mesh migration into the cecum following inguinal hernia repair. Gastroenterol Hepatol. 2009; 5: 361-364.
Austin J Surg - Volume 4 Issue 1 - 2017

ISSN : 2381-9030 | www.austinpublishing group.com

Kharief. () All rights are reserved
Citation: Kharief A. Mesh Migration in to Colonic Lumen Post Abdominal Hernia Repair: A Case Report. Austin J Surg. 2017; 4(1): 1095. 